\title{
Drought-tolerant sugarcane improves rhizosphere bacterial community in response to drought stress
}

\author{
Qi Liu ${ }^{1}$, Huichun Yang $^{1}$, Xiaowen Zhao ${ }^{1}$, Zixuan Wang ${ }^{1}$, Xindi Cui ${ }^{1}$, Ziting Wang ${ }^{1}$, \\ Muqing Zhang ${ }^{1}$, and Bao-Shan Chen ${ }^{1}$ \\ ${ }^{1}$ Guangxi University
}

April 28, 2020

\begin{abstract}
Aims Drought is one of the most important abiotic stresses currently threatening agricultural production. Compared to watersensitive plant varieties, drought-tolerant varieties have a higher adaptability to drought. Rhizosphere bacteria, which are essential for coordinating host plant health, are also severely affected by drought stress. The response to drought stress of rhizosphere bacterial communities from drought-tolerant plants has become a hot spot of current research. We aim to explore the effect of drought stress on the root microorganisms of sugarcane, and the response of rhizosphere microorganisms of droughttolerant varieties to drought stress. Methods We used pot experiments to test the effects of drought stress on two sugarcane varieties, ZZ9 (drought-tolerant) and GT39 (water-sensitive). Through high-throughput sequencing of bacterial communities in the rhizosphere and analysis of physiological and biochemical characteristics of the rhizosphere soil, changes in the bacterial communities of ZZ9 and GT39 due to drought stress were analyzed, and the relationship between the changes and the soil environment was studied. Conclusions Drought stress can reduce the diversity of bacterial communities in the rhizosphere of both plant varieties. Under drought stress, drought-tolerant varieties can better accumulate symbiotic bacteria to cope with the stressful conditions.
\end{abstract}

\section{Drought-tolerant sugarcane improves rhizosphere bacterial community in response to drought stress}

Qi Liua, b, c, Huichun Yanga, b, c, Xiaowen Zhao a, b, c , Zixuan Wanga, b, c, Xindi Cui ${ }^{a, b}$, c , Ziting Wanga, b, c*, Muqing Zhang, $\mathrm{b}, \mathrm{c}^{*}$, Baoshan Chen ${ }^{\mathrm{a}, \mathrm{b}, \mathrm{c}^{*}}$

a Guangxi Key Laboratory of Sugarcane Biology, Nanning, Guangxi 530004, PR China

b State Key Laboratory for Conservation \& Utilization of Subtropical Agro-bioresources, Guangxi University, Nanning, Guangxi 530004, PR China

c College of Agronomy, Guangxi University, Nanning, Guangxi 530004, PR China

${ }^{*}$ Corresponding author.

Ziting Wang, Muqing Zhang and Baoshan Chen are the common Corresponding authors.

E-mail addresses:zitingwang@gxu.edu.cn (Z.T.Wang), zmuqing@163.com (M.Q.Zhang) and chenyaoj@gxu.edu.cn(B.S. Chen)

Qi Liu and Huichun Yang are the common first authors.

\section{Hosted file}

Manuscript.docx available at https://authorea.com/users/313404/articles/443918-drought-tolerantsugarcane-improves-rhizosphere-bacterial-community-in-response-to-drought-stress 

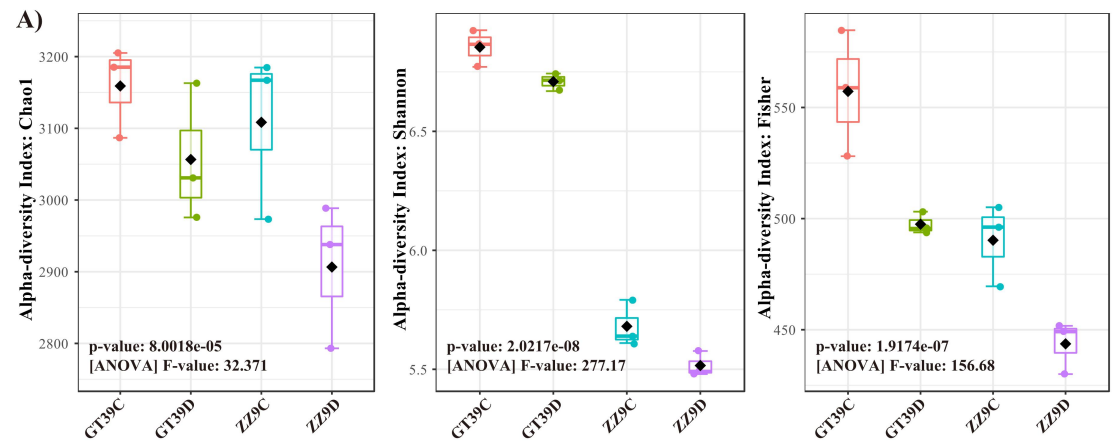

B)

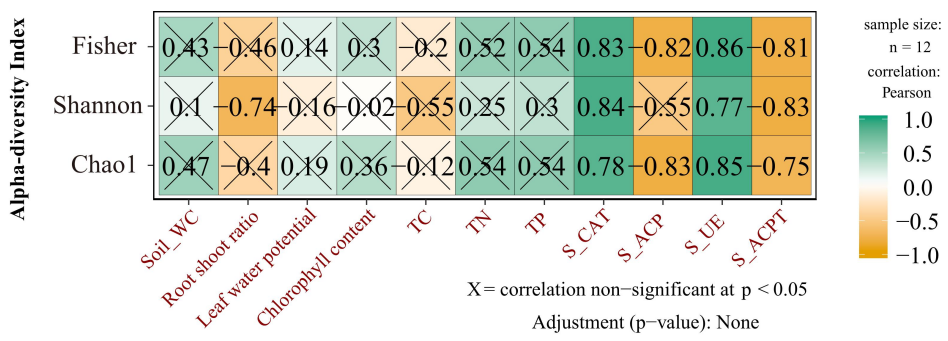

A)

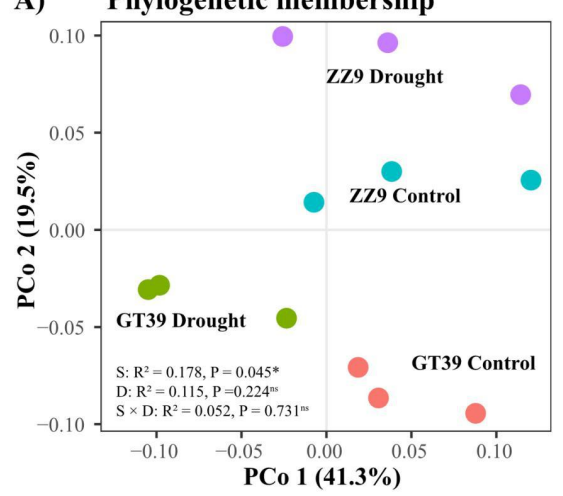

C)

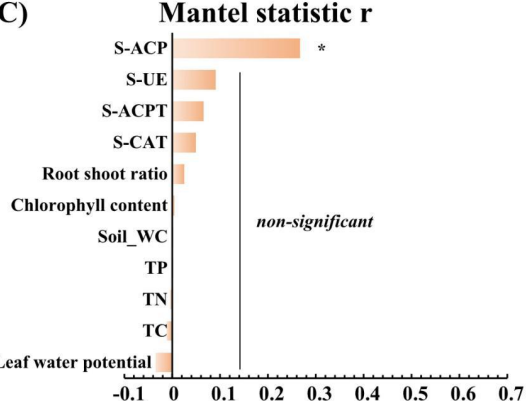

B) Phylogenetic compotion
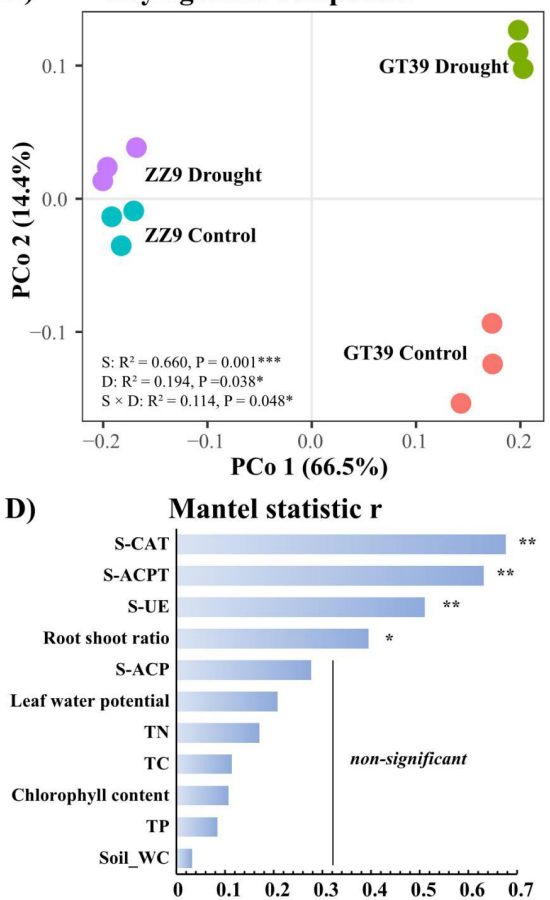

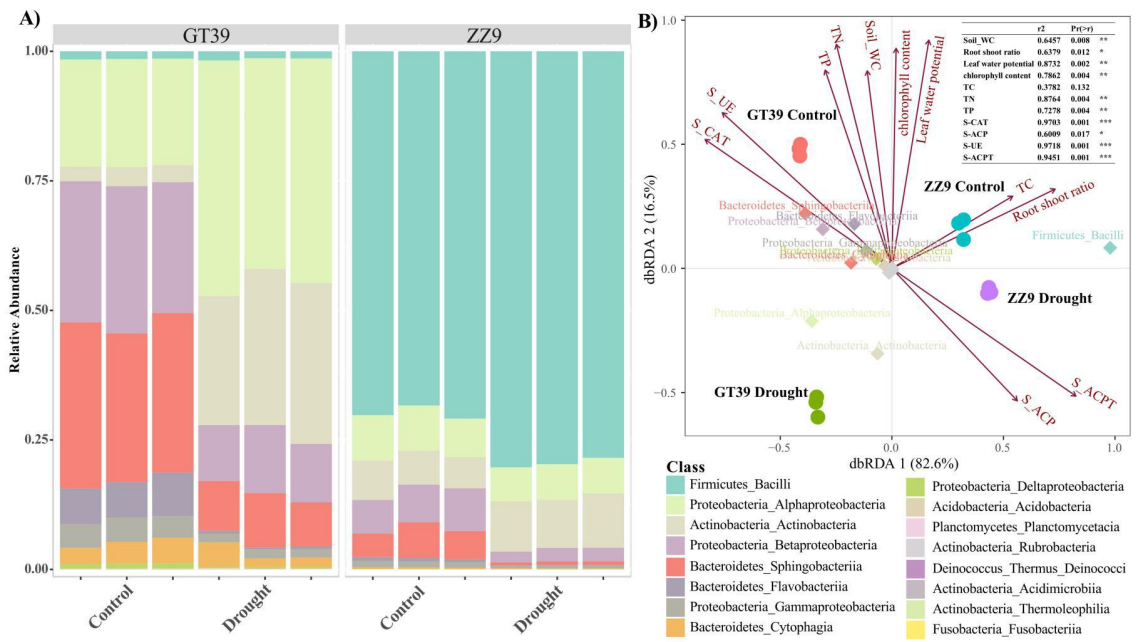

A)
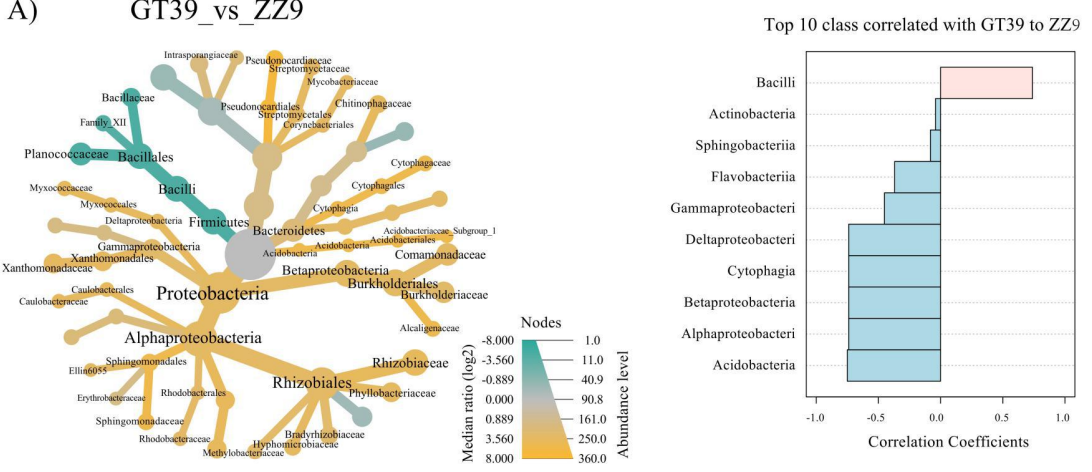

B) Control_vs_Drought
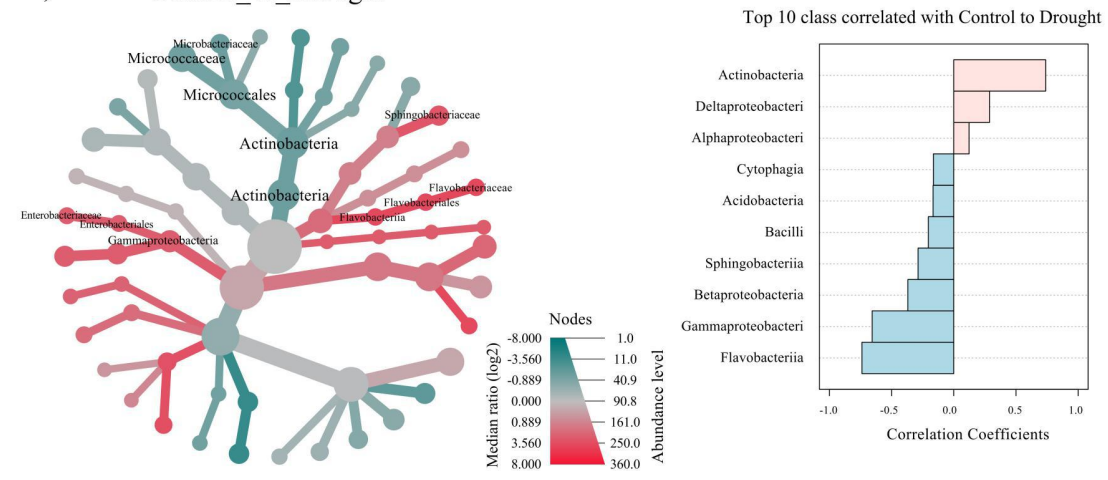

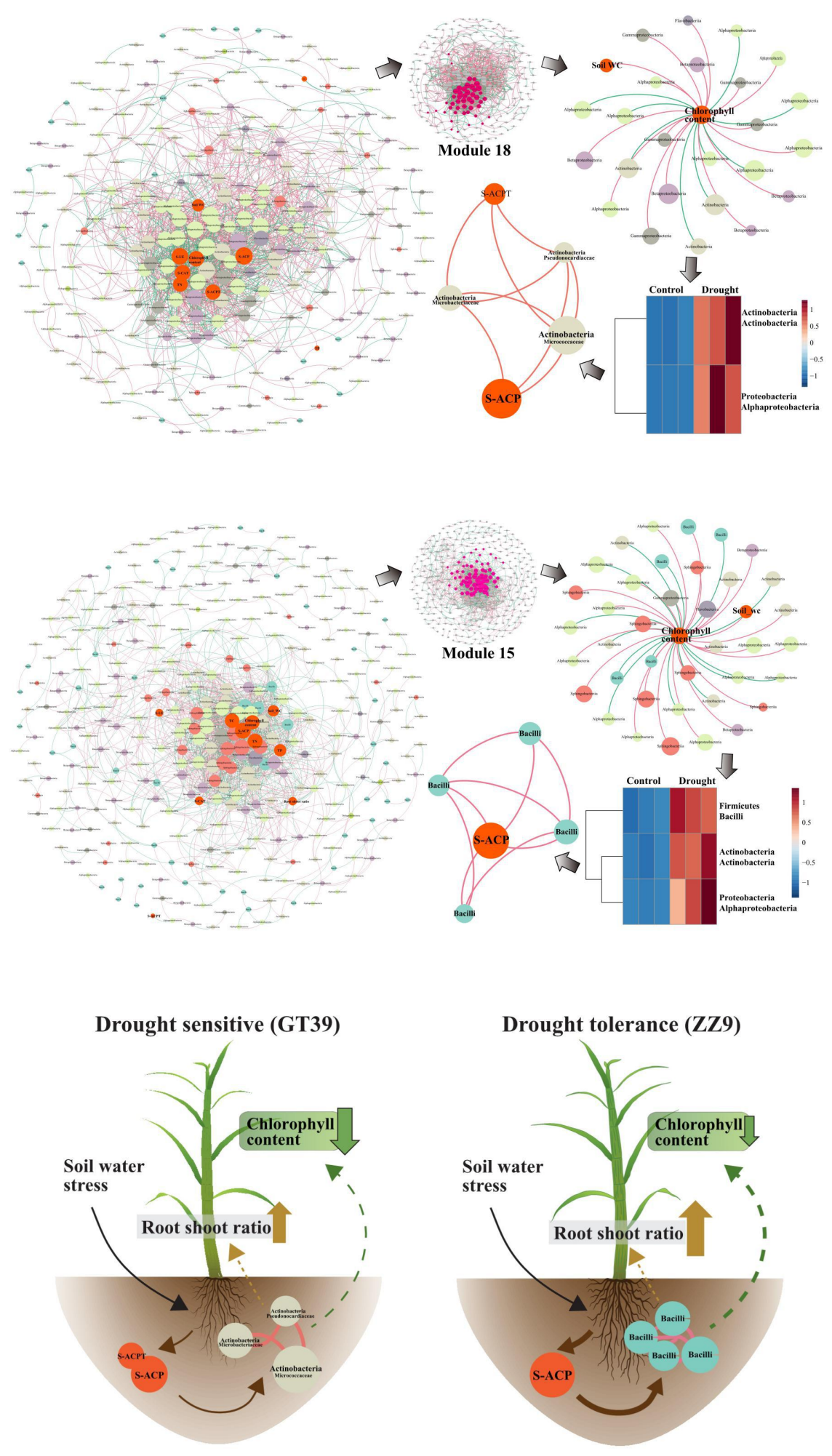